\title{
Article \\ Helminth Infections in Cattle and Goats in Kanchanaburi, Thailand, with Focus on Strongyle Nematode Infections
}

\author{
Nicharee Income ${ }^{1,2}$, Jarinee Tongshoob ${ }^{1}$, Sarawut Taksinoros ${ }^{3}$, Poom Adisakwattana ${ }^{4}$, \\ Chawarat Rotejanaprasert ${ }^{5,6} \oplus$, Pannamas Maneekan ${ }^{5}$ and Nathamon Kosoltanapiwat ${ }^{1, *}$ (]) \\ 1 Department of Microbiology and Immunology, Faculty of Tropical Medicine, Mahidol University, \\ 420/6 Ratchawithi Road, Ratchathewi, Bangkok 10400, Thailand; nicharee.inc@mahidol.ac.th (N.I.); \\ jarinee.pan@mahidol.ac.th (J.T.) \\ 2 Livestock and Wildlife Hospital, Mahidol University, 199 Lum Sum, Sai Yok, Kanchanaburi 71150, Thailand \\ 3 Department of Clinical Science and Public Health, Faculty of Veterinary Science, Mahidol University, \\ 999 Salaya, Phutthamonthon, Nakhon Pathom 73170, Thailand; sarawut.tak@mahidol.ac.th \\ 4 Department of Helminthology, Faculty of Tropical Medicine, Mahidol University, 420/6 Ratchawithi Road, \\ Ratchathewi, Bangkok 10400, Thailand; poom.adi@mahidol.ac.th \\ 5 Department of Tropical Hygiene, Faculty of Tropical Medicine, Mahidol University, 420/6 Ratchawithi Road, \\ Ratchathewi, Bangkok 10400, Thailand; chawarat.rot@mahidol.ac.th (C.R.); \\ pannamas.man@mahidol.ac.th (P.M.) \\ 6 Mahidol-Oxford Tropical Medicine Research Unit, Faculty of Tropical Medicine, Mahidol University, \\ 420/6 Ratchawithi Road, Ratchathewi, Bangkok 10400, Thailand \\ * Correspondence: nathamon.kos@mahidol.ac.th; Tel.: +662-306-9172
}

check for

updates

Citation: Income, N.; Tongshoob, J.; Taksinoros, S.; Adisakwattana, P.; Rotejanaprasert, C.; Maneekan, P.; Kosoltanapiwat, N. Helminth Infections in Cattle and Goats in Kanchanaburi, Thailand, with Focus on Strongyle Nematode Infections. Vet. Sci. 2021, 8, 324. https://doi.org/ $10.3390 /$ vetsci 8120324

Academic Editor: Michael D. Flythe

Received: 25 October 2021

Accepted: 7 December 2021

Published: 12 December 2021

Publisher's Note: MDPI stays neutral with regard to jurisdictional claims in published maps and institutional affiliations.

Copyright: (C) 2021 by the authors. Licensee MDPI, Basel, Switzerland. This article is an open access article distributed under the terms and conditions of the Creative Commons Attribution (CC BY) license (https:// creativecommons.org/licenses/by/ $4.0 /)$.
Abstract: Gastrointestinal helminths are major enteric parasites affecting the health of important livestock ruminants, such as cattle and goats. It is important to routinely survey these animals for helminth infections to allow effective management and control programs to be implemented. A cross-sectional helminth survey carried out in Kanchanaburi Province, Thailand, revealed the infection rate of gastrointestinal helminths in cattle $(n=157)$ and goats $(n=117)$ to be $35.7 \%$ and $88 \%$, respectively, by microscopic fecal examination, and a $100 \%$ herd prevalence was observed in goats. Eggs of strongyle nematodes, Strongyloides spp., Trichuris spp., Capillaria spp., Paramphistomum spp., and Moniezia spp. were detected, with a relatively high rate of strongyle nematode infection in both cattle (28.7\%) and goats (86.3\%). Mixed infections were observed in $14.3 \%$ and $35.9 \%$ of eggpositive samples from cattle and goats, respectively. Risk factor analysis showed that dairy cattle were 5.1 times more likely to be infected with strongyles than meat cattle. In contrast, meat goats were 9.3 times more likely to be infected with strongyles than dairy goats. The inverse findings in cattle and goats are discussed. Female gender was associated with a higher risk of strongyle infection in goats. DNA sequencing and in-house semi-nested PCR with primers specific to a region in the internal transcribed spacer 2 (ITS2) were successfully used to identify strongyle genera in randomly selected egg-positive cattle $(n=24)$ and goat $(n=24)$ samples. Four strongyle genera, i.e., Cooperia spp., Haemonchus spp., Oesophagostomum spp., and Trichostrongylus spp. were identified by DNA sequencing. By semi-nested PCR, Cooperia spp. were detected as a major parasite of cattle $(70.8 \%)$, whereas Haemonchus spp. were abundant in goats $(100 \%)$. The majority of samples from cattle $(58.3 \%)$ and goats $(95.8 \%)$ were found to coinfect with at least two strongyle genera, suggesting that coinfection with multiple strongyle genera was more common than single infection in these animals.

Keywords: cattle; goat; helminth infection; strongyle nematode; risk factor; PCR

\section{Introduction}

Gastrointestinal helminths are one of the most important disease-causing agents in veterinary medicine, especially in livestock, and lead to economic losses as a result of a decrease in meat, milk, or wool production [1,2]. Most gastrointestinal helminths infect 
animals via the ingestion of infective-stage larvae or eggs. The eggs and larvae are excreted with the host's feces into the environment and become a source of transmission. Strongyle nematodes of the order Strongylida are an important group of gastrointestinal helminths that significantly affect the health of ruminants, especially in tropical areas [3-5].

In Thailand, a prevalence of strongyle infections (27\%) was reported in beef cattle from Nan Province in 2005 [6]. Those cattle were raised in a native pasture grazing system with poor sanitation and a lack of deworming. A high strongyle infection rate $(79.47 \%)$, with $100 \%$ herd prevalence, was found among goats in Nakhon Pathom Province, Thailand, in 2010 [7]. The study showed that the housing system, deworming interval, and type of goats in the herd were risk factors for intestinal parasite infection. Surveys conducted in other Southeast Asian countries also point to the importance of strongyle infections in ruminants. In Lao PDR, strongyles were detected in 36\% of cattle and $93 \%$ of goat fecal samples collected in 2010 [3]. Trichostrongylus spp., Haemonchus spp., and Oesophagostomum spp. were reported in cattle and goats, while Cooperia spp. were identified in cattle. Trichostrongylus spp. and Haemonchus spp. were found to be dominant parasites in cattle and goats, respectively. A report from Malaysia in 2014 described the incidence of strongyle infection in cattle $(11.2 \%)$, and goats (63.1\%) [4]. In the same study, a coinfection of two strongyles, Haemonchus spp. and Trichostrongylus spp., was detected in goat feces by amplification and sequencing of the internal transcribed spacer 2 (ITS2) DNA region. Furthermore, $100 \%$ herd prevalence and $96.22 \%$ animal-level prevalence of strongyles were reported in goats raised in the Philippines in 2016 [8], and the strongyle genera detected were Haemonchus spp., Trichostrongylus spp., Oesophagostomum spp., Cooperia spp., and Chabertia spp. Evidence of strongyle nematode infections in ruminants has been consistently found in South Asia, Africa, and Europe until the present day [9-13]. Among these, anthelmintic drug resistance was widely reported in goats infected with Haemonchus spp. [12,13]. For decades, benzimidazoles such as albendazole, imidothiazoles-tetrahydropyrimidines, and avermectin have been the major anthelmintic classes used for treating gastrointestinal nematodes of small ruminants. However, recent reports have shown a growing resistance to these drugs both in vitro and in vivo [12-15]. Factors contributing to the development of drug resistance could be mixed grazing of difference animal species such as goats and sheep, using under-dosage of anthelmintics, and using the same group of drugs for a long time $[15,16]$. Besides strongyles, other nematodes, such as Trichuris spp., as well as cestodes, such as Moniezia spp., and rumen flukes (trematodes), such as Paramphistomum spp., have been found to cause less severe problems in ruminants. Eggs of Moniezia spp. were detected in the feces of cattle, goats, and sheep in Vietnam [17] and goats in Thailand [7], and rumen fluke have been reported to infect beef cattle in Thailand, with no seriously observable clinical signs [6].

For diagnosis of veterinary helminth infections in Thailand, a microscopic stool examination is routinely performed. The technique provides a wide range of parasite detection but barely identifies the parasite at genus and species level. Molecular identification by PCR is considered to be a rapid, uncomplicated, and cost-effective method with high sensitivity and specificity that has enabled the accurate identification of parasite species $[4,17]$. The genomic regions widely used in the molecular detection and identification of the parasitic helminths are ITS of ribosomal DNA (rDNA), which in eukaryotes features non-transcribed spacer (NTS), external transcribed spacer (ETS), 18S rDNA, ITS1, 5.8S rDNA, ITS2, and $28 \mathrm{~S}$ rDNA $[4,17,18]$. Coding regions of rDNA are highly conserved among species and, hence, are appropriate for pan-strongyle primer design, whereas ITS regions are more variable because of insertions, deletions, and point mutations. The ITS2 sequence has been utilized for strongyle identification owing to its high intraspecific sequence homogeneity and high interspecific sequence divergence [4]. Analysis of ITS2 has previously been used to confirm morphological diagnoses of Trichostrongylus spp., Oesophagostomum spp., and Haemonchus spp. [3].

Due to a lack of published information concerning helminthic infections in cattle and goats in Kanchanaburi Province, Thailand, the prevalence of gastrointestinal helminthic 
infection in cattle and goats was surveyed by egg microscopic examination. As the animals were found to be infected with strongyle nematodes at a high rate, the strongyles subsequently became the main focus of the study. Risk factors associated with strongyle infection were investigated. ITS2 sequencing was used to identify strongyle genera that were circulating in cattle and goats. In-house semi-nested PCR was used to differentiate strongyles to the genus level and ascertain the coinfection status of animals.

\section{Materials and Methods}

\subsection{Sample and Data Collection}

Fecal and blood samples, and animal information were collected from 157 cattle and 117 goats in 11 cattle farms and 9 goat farms, respectively, located in seven districts of Kanchanaburi Province in the western part of Thailand, in May-July 2016 (Figure 1). A cross-sectional survey was performed, simultaneously collecting samples and relevant demographic and management data during a single farm visit. Random sampling was conducted in farms that allowed our visit. The cattle farms included 10 intensive management farms, where cattle were restricted to the farm area and fed concentrated food and roughage, such as grass, hay, or corn, and one extensive management farm, where cattle were released to graze freely during the day and were housed indoors at night. The goat farms included eight extensive management farms and one intensive management farm, where goats were housed in a cage and fed with concentrated food and roughage, including grass and leaves. Each farm housed 15 to 50 animals.

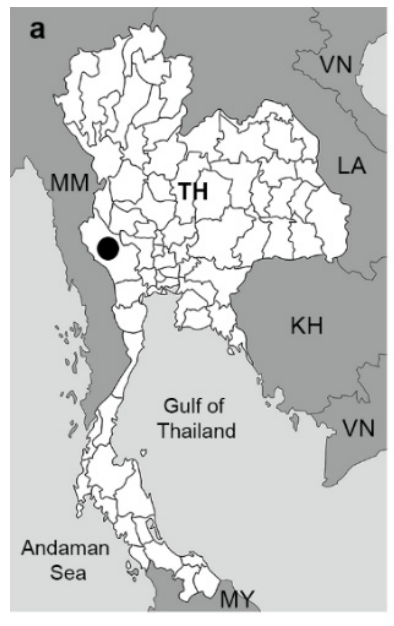

b

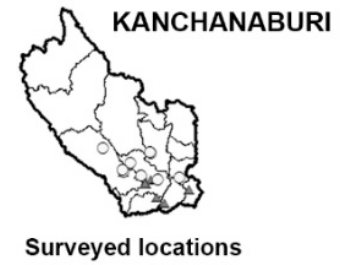

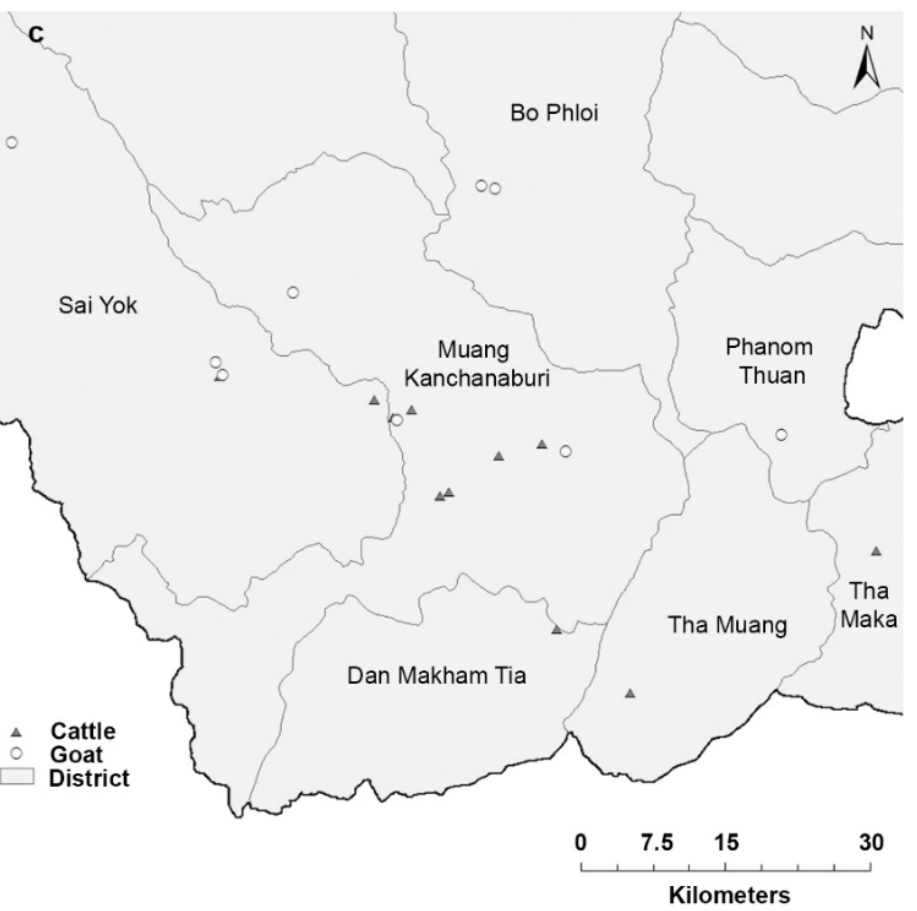

Figure 1. Map showing the sample collection sites in Kanchanaburi, Thailand. (a) Thailand map with Province outline — the black circle indicates Kanchanaburi Province. TH, Thailand; KH, Cambodia; LA, Lao People's Democratic Republic; MM, Myanmar; MY, Malaysia; VN, Vietnam. (b,c) Surveyed locations in Kanchanaburi. Triangles represent the cattle farms (11 farms), and circles represent the goat farms (9 farms). The names of the districts in Kanchanaburi Province are shown on the map. Images in $(\mathbf{b}, \mathbf{c})$ were adapted from the authors' published work. (Reprinted with permission from Income, N.; Kosoltanapiwat, N.; Taksinoros, S.; Leaungwutiwong, P.; Maneekan, P.; Chavez, I.F. (2019). Copyright @ 2019 American Society for Microbiology, Appl. Environ. Microbiol. 85: e02420-18 doi: 10.1128/AEM.02420-18.). 
Fecal samples were directly collected from the animal rectum using clean gloves. Whole blood was collected from the tail vein (cattle) or jugular vein (goat), preserved in an EDTA tube, and sent to the Hematology Laboratory, Livestock and Wildlife Hospital, Faculty of Veterinary Science, Mahidol University, Kanchanaburi, within $24 \mathrm{~h}$. The packed cell volume (PCV) was determined by microhematocrit centrifugation using the EDTAwhole blood and categorized as anemic (PCV $<22 \%$ in goat, PCV $<24 \%$ in cattle) or non-anemic (PCV $\geq 22 \%$ in goat, $\mathrm{PCV} \geq 24 \%$ in cattle) [19].

Animal identification (name and number), gender, age, production purpose (dairy or meat), farm management (intensive or extensive management), and deworming interval were recorded. Animals were grouped as young ( $<1$ year) and adult ( 1 year and above). Body condition (thin to obese) was scored for cattle [20] and goats [21]. Oral mucosa color was observed and recorded as pink, pale pink or pale. All examinations were performed by a trained veterinarian.

\subsection{Microscopic Examination}

Microscopic examination of fecal samples was conducted using simple flotation and formalin-ether concentration techniques.

Flotation technique: Approximately $2 \mathrm{~g}$ of feces were mixed with $10 \mathrm{~mL}$ of saturated $\mathrm{NaCl}$ and filtered through two layers of wet cotton gauze into a $15 \mathrm{~mL}$ conical tube. Additional saturated $\mathrm{NaCl}$ was added to fill the tube, leaving a convex meniscus at the top, then a coverslip was carefully placed over the opening of the test tube. After leaving the test tube to stand for 10-15 min, the coverslip was carefully and vertically removed from the tube, immediately placed on a microscope slide, and examined under a light microscope.

Formalin-ether concentration technique: Approximately $2 \mathrm{~g}$ of feces were mixed with $10 \mathrm{~mL}$ of $10 \%$ formalin and filtered through two layers of wet cotton gauze into a $15 \mathrm{~mL}$ conical tube. Additional $10 \%$ formalin was added to adjust to a total volume of $10 \mathrm{~mL}$, followed by the addition of $2 \mathrm{~mL}$ of ether. The liquid was shaken vigorously and centrifuged at $400 \times g$ for $2 \mathrm{~min}$. Four layers became visible: a top layer of ether, a second debris plug layer, a third clear layer of formalin, and a fourth layer of sediment. The debris plug was detached from the side of the tube using a small stick. The layers of ether, debris plug, and formalin were poured off to leave a small amount of formalin and sediment (approximately $2 \mathrm{~mL}$ ). The tube was left undisturbed for $10 \mathrm{~min}$ to allow the precipitate to form. The residual liquid was removed by pipetting, leaving about $0.5 \mathrm{~mL}$ of formalin. The precipitate and formalin were mixed and dropped onto a slide, covered with a coverslip, and examined under a light microscope.

Helminth egg identification and semi-quantification were performed according to the parasitology laboratory manual used at the Livestock and Wildlife Hospital, Mahidol University, Thailand. Egg burdens were graded at levels of 1-4. Grade 1, 2, 3, and 4 refer to $1-4,5-8,9-12$, and 13 or more eggs per slide, respectively. Samples with at least grade 1 egg burden were considered as a positive sample.

\subsection{Fecal Sample Preparation and DNA Extraction}

Approximately $2 \mathrm{~g}$ of feces were mixed with $10 \mathrm{~mL}$ of $2 \%$ dish-washing soap solution and filtered through two layers of wet cotton gauze into a 15-mL conical tube. The tube was centrifuged at $1500 \times g$ for $10 \mathrm{~min}$, and the liquid was discarded. The pellet was resuspended in $10 \mathrm{~mL}$ of sterile water, and the steps of filtration, centrifugation, and pellet dissolution were repeated twice. After the three rounds of filtering and centrifugation, the fecal pellet was resuspended in $0.5 \mathrm{~mL}$ sterile water, transferred to a microcentrifuge tube, and centrifuged at $5500 \times g$ for $5 \mathrm{~min}$. The liquid was totally removed by pipetting, and the pellet was stored at $-80^{\circ} \mathrm{C}$ for subsequent DNA extraction.

The processed fecal pellet was snap-frozen in liquid nitrogen and ground immediately using a disposable pestle for three cycles. Subsequently, the sample was vortex-mixed in $1.4 \mathrm{~mL}$ of buffer ASL provided in the QIAamp DNA stool mini kit (Qiagen, Hilden, Germany) and transferred into a 2-mL sample tube RB (Qiagen, cat. no. 990381). A sterile 
stainless-steel bead, $5 \mathrm{~mm}$ in diameter (Qiagen, cat. no. 69989), was added to the tube, and the sample was homogenized using TissueLyser LT (Qiagen) at $50 \mathrm{~Hz}$ for $5 \mathrm{~min}$, twice. The homogenized sample was subjected to DNA extraction using the QIAamp DNA Stool Mini Kit according to the manufacturer's instructions. DNA was eluted with $50 \mu \mathrm{L}$ of $\mathrm{AE}$ elution buffer and stored at $-20^{\circ} \mathrm{C}$.

\subsection{Semi-Nested PCR for Detection and Differentiation of Strongyles}

To detect the presence of strongyle helminths in the fecal samples, PCR was performed with primers specific to regions of strongyle nematode ribosomal DNA and ITS2 (Table 1). The PCR reaction contained $1 \times$ MyTaq HS Red Mix (Bioline, London, UK), $0.2 \mu \mathrm{M}$ of each forward (Strongyle F2) and reverse (Strongyle R3) primer, and $1 \mu \mathrm{L}$ of DNA template in a total volume of $25 \mu \mathrm{L}$. The thermal cycling conditions were set as $95^{\circ} \mathrm{C}$ for $3 \mathrm{~min}$, followed by 35 cycles of $95{ }^{\circ} \mathrm{C}$ for $30 \mathrm{~s}, 50{ }^{\circ} \mathrm{C}$ for $30 \mathrm{~s}$, and $72{ }^{\circ} \mathrm{C}$ for $30 \mathrm{~s}$, and a final elongation at $72{ }^{\circ} \mathrm{C}$ for $10 \mathrm{~min}$. PCR products were resolved and visualized by agarose gel electrophoresis, and sent for DNA sequencing.

Table 1. Primer sequences for strongyle detection by semi-nested PCR.

\begin{tabular}{cccc}
\hline Primer & Sequence $\left(\mathbf{5}^{\prime}-\mathbf{3}^{\prime}\right)$ & Region & Product Size (bp) $^{\mathbf{1}}$ \\
\hline Strongyle F2 & TGGTGAAATTTTGAACGCATAG & 5.8 S rRNA & - \\
Strongyle R3 & ATGCTTAAGTTCAGCGGGTA & 28S rRNA & $324-349$ \\
Cooper R & CGAATACTACTATCTCCAACATG & ITS2 & 293 \\
Haemo R & GTACACTCAAATAGWGGCAACAT & ITS2 & 227 \\
Oeso R & CTCATCTAGAACGAGGATCACA & ITS2 & 143 \\
Tricho R & CAATATTTGAYAATGACCATTCG & ITS2 & 128 \\
\hline
\end{tabular}

${ }^{1}$ PCR product sizes were estimated from alignment of each primer with representatives of strongyle genera (GenBank: MH267779.1, AB908961.1, HQ844232.1, and AB908960.1).

In-house semi-nested PCR primers were used to detect and differentiate the four strongyle genera that were found in this study, i.e., Cooperia spp., Haemonchus spp., Oesophagostomum spp., and Trichostrongylus spp. Primer sequences and expected PCR product sizes are shown in Table 1. Alignments of strongyle sequences and the specificity of the primers are shown in the Supplemental Figures S1 and S2. The semi-nested PCR reaction and PCR thermal cycling conditions were set up as mentioned above, except that the reverse primer was replaced with the genus-specific primer and $1 \mu \mathrm{L}$ of diluted F2/R3 PCR product (1:100 in nuclease-free water) was used as the template.

\subsection{Nucleotide Sequencing and Phylogenetic Analysis}

PCR products of the expected size were excised from an agarose gel. The DNA was purified using a QIAquick gel extraction kit (Qiagen) following the manufacturer's instructions. Purified PCR products were sent for nucleotide sequencing (Bioneer, Daejeon, Korea) using F2 primers. Sequencing chromatograms were inspected and processed using BioEdit 7.0.4.1. Nucleotide sequences were queried against the NCBI database using the BLAST tool and aligned with published reference sequences using ClustalW in BioEdit. A total of 14 strongyle sequences were submitted to the GenBank database and received the accession numbers MT294426, MT294427, MT294428, MT294429, MT294430, MT294431, MT294432, MT294433, MT294434, MT294435, MT294436, MT294437, MT294438, and MT294439. A phylogenetic tree was constructed using the maximum likelihood method based on the Kimura 2-parameter model and bootstrap resampling analysis of 1000 replicates in MEGA 7.0.21.

\subsection{Statistical Analysis}

The infection rate of strongyles and corresponding 95\% confidence intervals (CI) were calculated using the Wilson approximation method. The univariable analysis by Chi-squared test was performed to evaluate risk factors at a $5 \%$ level of significance $(p<0.05)$ using the status of strongyle infection as the dependent variable and the animal 
and management factors (gender, age, body condition score, mucous membrane, PCV categories, production purpose, farm management, deworming interval, and flocks) as the independent variables. Significant variables $(p<0.05)$ in the univariable analysis were used to perform a backward stepwise multivariable logistic regression model to calculate the adjusted odds ratios (AOR) at $5 \%$ level of significance. Data analyses were conducted using RStudio software version 1.4.1717.

\section{Results}

3.1. Detection of Gastrointestinal Helminth Eggs in Cattle and Goat Feces by Microscopic Examination

Fecal samples were collected from 11 cattle farms and 9 goat farms located in seven districts of Kanchanaburi Province, Thailand, in 2016 (Figure 1). The presence of helminth eggs in all cattle $(n=157)$ and goat $(n=117)$ fecal samples was qualitatively examined under a microscope after processing the samples with simple flotation and formalin-ether concentration techniques. The eggs of strongyle nematodes, Strongyloides spp., Trichuris spp., Capillaria spp., Paramphistomum spp., and Moniezia spp. were detected (Figure 2). Gastrointestinal helminth eggs were detected in $35.7 \%$ of cattle samples and $88 \%$ of goat samples. At the farm level, helminth egg-positive samples were found in 10 of the 11 cattle farms $(90.9 \%$ herd prevalence) and in all nine goat farms (100\% herd prevalence). The numbers and percentages of samples containing helminth eggs are shown in Table 2. Mixed infections of more than one type of helminth were found in $14.3 \%(8 / 56)$ and $35.9 \%(37 / 103)$ of egg-positive cattle and goat samples, respectively. Strongyle nematode eggs were most the abundant egg type in both cattle $(28.7 \%)$ and goats $(86.3 \%)$.
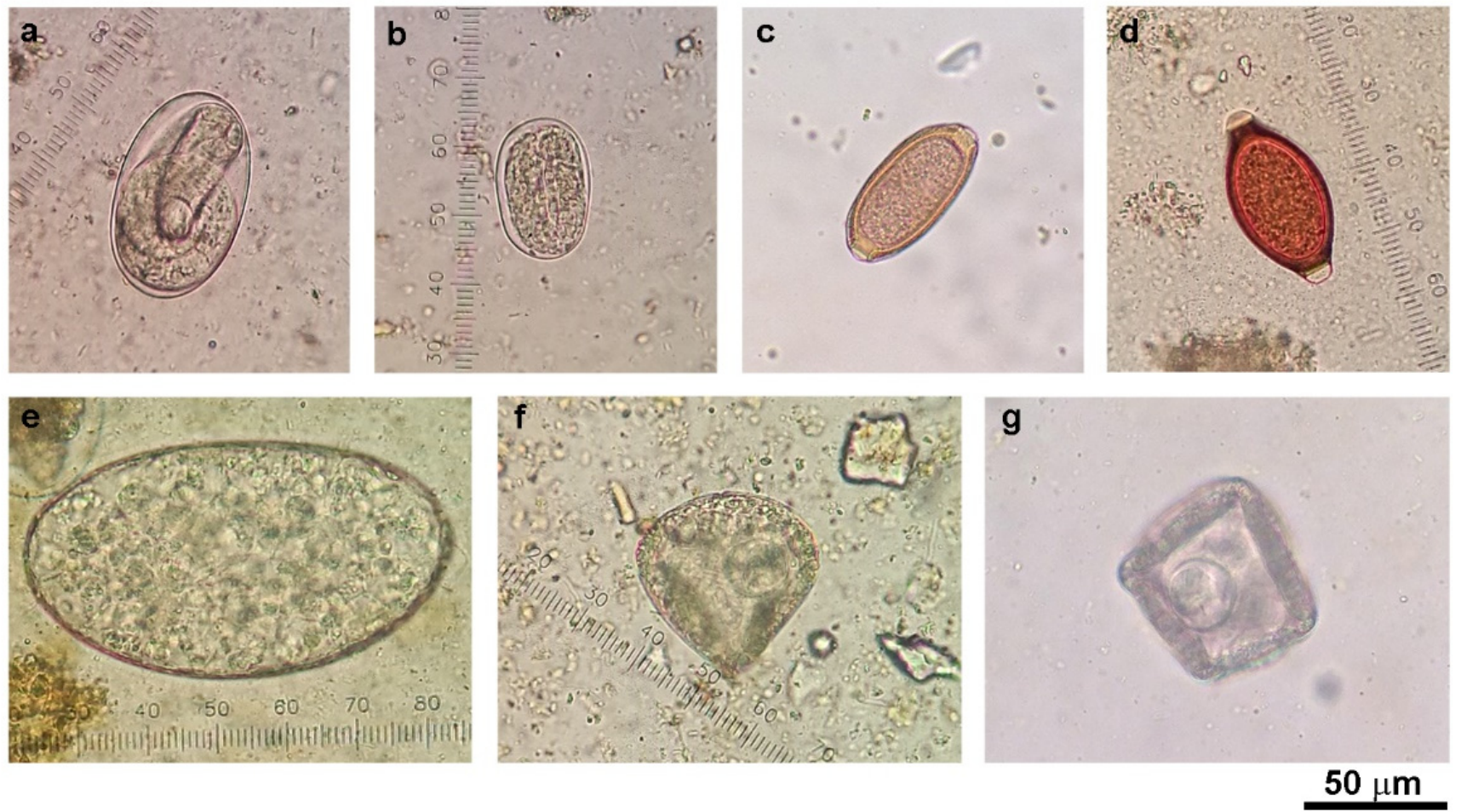

Figure 2. Morphology of gastrointestinal helminth eggs detected in cattle and goat feces by microscopic examination: (a) Strongyle egg (larvated egg); (b) Strongyloides spp. egg (larvated egg); (c) Capillaria spp. egg; (d) Trichuris spp. egg; (e) Paramphistomum spp. egg; (f) Moniezia spp. egg from goat; and (g) Moniezia spp. egg from cattle. Photos were taken under $400 \times$ magnification. 
Table 2. Survey of gastrointestinal helminth eggs by microscopic examination.

\begin{tabular}{ccc}
\hline \multirow{2}{*}{ Helminth Species } & \multicolumn{2}{c}{ No. of Helminth Egg Positive Samples (\%) } \\
\cline { 2 - 3 } & Cattle $(\boldsymbol{n = 1 5 7 )}$ & Goat $(\boldsymbol{n = 1 1 7})$ \\
\hline Strongyle nematodes & $45(28.7)$ & $101(86.3)$ \\
Strongyloides spp. & $1(0.6)$ & $18(15.4)$ \\
Trichuris spp. & $0(0)$ & $7(6)$ \\
Capillaria spp. & $1(0.6)$ & $5(4.3)$ \\
Paramphistomum spp. $_{\text {Moniezia spp. }}$ & $16(10.2)$ & $9(7.7)$ \\
Total $^{1}$ & $1(0.6)$ & $5(4.3)$ \\
\hline
\end{tabular}

${ }^{1}$ Total number of fecal samples that were helminth egg-positive. Some samples contained more than one type of egg.

3.2. Infection Rate, Egg Burden and Risk Factors of Strongyle Infection among Cattle and Goats in Kanchanaburi Province

We further focused on analyses of strongyle infection as the parasite was abundantly detected in cattle and goats. The overall infection rate and 95\% CI for strongyle infection based on the risk factor categories is shown in Table 3 for cattle and Table 4 for goats. Strongyle egg-positive samples were found in nine of the 11 cattle farms $(81.8 \%$ herd prevalence, $95 \% \mathrm{CI}=47.75-96.78 \%)$, and in eight of the nine goat farms $(88.9 \%$ herd prevalence, $95 \% \mathrm{CI}=50.67-99.42 \%)$. Egg burden was determined by semi-quantitative grading of the egg number. As shown in Figure 3, the majority of the strongyle egg-positive cattle $(66.7 \%)$ were lightly infected $(1+)$, whereas most of the strongyle egg-positive goats $(74.3 \%)$ were heavily infected $(4+)$.

Table 3. The overall infection rate and univariable analysis for risk factors of strongyle infection in cattle.

\begin{tabular}{|c|c|c|c|c|c|}
\hline Factor & Number & Infection Rate (\%) & $95 \% \mathrm{CI}$ & $x^{2}$ & $p$-Value \\
\hline \multicolumn{6}{|l|}{ Gender } \\
\hline Male & 4 & $1(25.0)$ & $4.55-69.93$ & 0.0269 & 0.8697 \\
\hline $\begin{array}{l}\text { Female } \\
\text { Age }\end{array}$ & 153 & $44(28.8)$ & $22.17-36.38$ & - & - \\
\hline Young & 0 & $0(0)$ & - & - & - \\
\hline Adult & 157 & $45(28.7)$ & $22.16-36.17$ & - & - \\
\hline \multicolumn{6}{|l|}{$\begin{array}{l}\text { condition } \\
\text { score }\end{array}$} \\
\hline Fat & 22 & $7(31.8)$ & $16.36-52.68$ & 0.481 & 0.786 \\
\hline Average & 117 & $34(29.1)$ & $21.60-37.84$ & - & - \\
\hline Thin & 18 & $4(22.2)$ & $9.01-45.21$ & - & - \\
\hline \multicolumn{6}{|l|}{$\begin{array}{l}\text { Oral mucosa } \\
\text { color }\end{array}$} \\
\hline Pink & 85 & $29(34.1)$ & $24.92-44.68$ & 2.886 & 0.2361 \\
\hline Pale pink & 69 & $15(21.7)$ & $13.64-32.81$ & - & - \\
\hline Pale & 3 & $1(33.3)$ & $6.14-79.23$ & - & - \\
\hline \multicolumn{6}{|l|}{$\begin{array}{c}\text { PCV } \\
\text { categories }\end{array}$} \\
\hline Non-anemic & 131 & $40(30.5)$ & $23.29-38.88$ & 1.356 & 0.244 \\
\hline Anemic & 26 & $5(19.2)$ & $8.51-37.87$ & - & - \\
\hline \multicolumn{6}{|l|}{$\begin{array}{l}\text { Production } \\
\text { purpose }\end{array}$} \\
\hline Dairy & 125 & $43(34.4)$ & $26.64-43.08$ & 9.873 & 0.002 * \\
\hline Meat & 32 & $2(6.3)$ & 1.73-20.14 & - & - \\
\hline
\end{tabular}


Table 3. Cont.

\begin{tabular}{cccccc}
\hline Factor & Number & Infection Rate (\%) & $\mathbf{9 5 \% ~ C I ~}$ & $\chi^{\mathbf{2}}$ & $p$-Value \\
\hline $\begin{array}{c}\text { Management } \\
\text { Intensive }\end{array}$ & 141 & $44(31.2)$ & $24.14-39.26$ & 4.377 & $0.036^{*}$ \\
Extensive & 16 & $1(6.3)$ & $0.28-8.71$ & - & - \\
Deworming & & & & \\
interval & & & & & \\
$\leq 6$ months & 30 & $0(0)$ & $0.00-11.35$ & 14.901 & $<0.001^{*}$ \\
$>6$ months & 127 & $45(35.4)$ & $27.65-44.06$ & - & - \\
a Flocks & & & & & $-0.001^{*}$ \\
A & 16 & $1(6.3)$ & $1.11-28.32$ & 34.499 & - \\
B & 1 & $1(100)$ & $20.65-100.00$ & - & - \\
C & 14 & $6(42.9)$ & $21.38-67.41$ & - & - \\
D & 15 & $7(46.7)$ & $24.81-69.88$ & - & - \\
E & 15 & $2(13.3)$ & $3.73-37.88$ & - & - \\
F & 13 & $8(61.5)$ & $35.52-82.29$ & - & - \\
G & 13 & $6(46.2)$ & $23.21-70.86$ & - & - \\
H & 15 & $0(0)$ & $0.00-20.39$ & - & - \\
I & 20 & $6(30)$ & $14.55-51.89$ & - & - \\
J & 20 & $8(40)$ & $21.88-61.34$ & - & - \\
K & 15 & $0(0)$ & $0.00-20.39$ & - & - \\
\hline Overall & 157 & $45(28.7)$ & $22.16-36.18$ & - &
\end{tabular}

* Statistically significant at $p<0.05 .{ }^{\text {a }}$ Letters A-K represent cattle flocks.

Table 4. The overall infection rate and univariable analysis for risk factors of strongyle infection in goats.

\begin{tabular}{|c|c|c|c|c|c|}
\hline Factor & Number & Infection Rate (\%) & $95 \% \mathrm{CI}$ & $x^{2}$ & $p$-Value \\
\hline \multicolumn{6}{|l|}{ Gender } \\
\hline Male & 18 & $11(61.1)$ & $38.61-79.69$ & 11.456 & $<0.001$ * \\
\hline Female & 99 & $90(90.9)$ & $83.61-95.14$ & - & - \\
\hline \multicolumn{6}{|l|}{ Age } \\
\hline Young & 5 & $4(80)$ & $37.55-96.37$ & 0.177 & 0.674 \\
\hline Adult & 112 & $97(86.6)$ & 79.07-91.71 & - & - \\
\hline \multirow{2}{*}{\multicolumn{6}{|c|}{ Body condition }} \\
\hline score & & & & & \\
\hline Fat & 15 & $12(80)$ & 54.81-92.95 & 4.006 & 0.134 \\
\hline Average & 82 & $69(84.1)$ & $74.74-90.49$ & - & - \\
\hline Thin & 20 & $20(100)$ & $83.88-100.00$ & - & - \\
\hline \multicolumn{6}{|c|}{ Oral mucosa color } \\
\hline Pink & 37 & $26(70.3)$ & $54.21-82.51$ & 11.848 & $0.003 *$ \\
\hline Pale pink & 79 & $74(93.7)$ & $86.02-97.26$ & - & - \\
\hline Pale & 1 & $1(100)$ & $20.65-100.00$ & - & - \\
\hline \multicolumn{6}{|c|}{ PCV categories } \\
\hline Non-anemic & 98 & $83(84.7)$ & 76.27-90.49 & 1.3597 & 0.243 \\
\hline Anemic & 19 & $18(94.7)$ & 75.36-99.06 & - & - \\
\hline $\begin{array}{l}\text { Production } \\
\text { purpose }\end{array}$ & & & & - & - \\
\hline Dairy & 20 & $11(55)$ & $34.20-74.18$ & 20.052 & $<0.001$ * \\
\hline Meat & 97 & $90(92.8)$ & $85.84-96.46$ & - & - \\
\hline \multicolumn{6}{|l|}{ Management } \\
\hline Intensive & 18 & $11(61.1)$ & $38.61-79.69$ & 11.456 & $<0.001$ * \\
\hline Extensive & 99 & $90(90.9)$ & 83.61-95.14 & - & - \\
\hline \multicolumn{6}{|c|}{ Deworming interval } \\
\hline$\leq 6$ months & 18 & $11(61.1)$ & $38.61-79.69$ & 11.456 & $<0.001 *$ \\
\hline$>6$ months & 99 & $90(90.9)$ & $83.61-95.14$ & - & - \\
\hline
\end{tabular}


Table 4. Cont.

\begin{tabular}{cccccc}
\hline Factor & Number & Infection Rate (\%) & $\mathbf{9 5 \%} \mathbf{C I}$ & $\chi^{\mathbf{2}}$ & $p$-Value \\
\hline a Flocks & & & & & \\
a & 18 & $11(61.1)$ & $38.61-79.69$ & 32.197 & $<0.001$ * \\
b & 15 & $14(93.3)$ & $70.18-98.81$ & - & - \\
c & 15 & $14(93.3)$ & $70.18-98.81$ & - & - \\
d & 15 & $11(73.3)$ & $48.04-89.10$ & - & - \\
e & 19 & $19(100)$ & $83.18-100.00$ & - & - \\
f & 2 & $0(0)$ & $0.00-65.76$ & - & - \\
g & 15 & $14(93.3)$ & $70.18-98.81$ & - & - \\
h & 3 & $3(100)$ & $43.85-100.00$ & - & - \\
i & 15 & $15(100)$ & $79.61-100.00$ & - & - \\
\hline Overall & 117 & $101(86.3)$ & $78.93-91.40$ & - & - \\
\hline
\end{tabular}

* Statistically significant at $p<0.05 .{ }^{\text {a }}$ Letters a-i represent goat flocks.

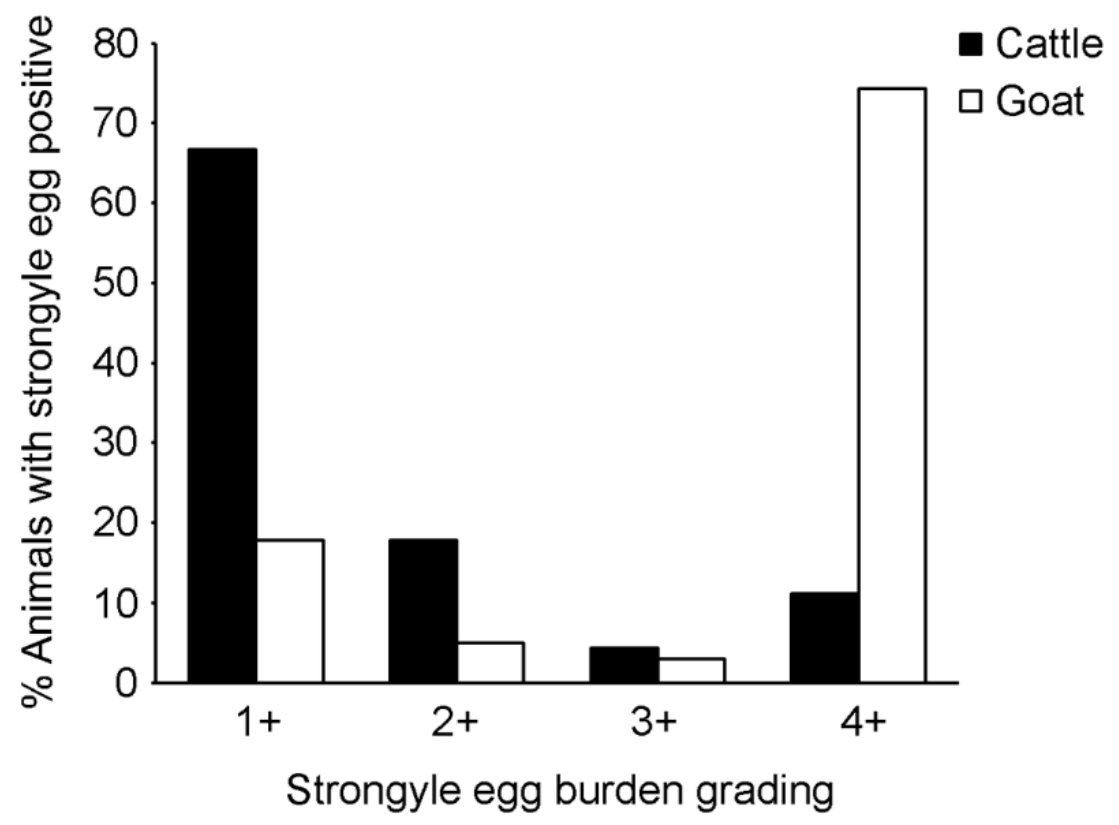

Figure 3. Strongyle egg burden grading. Strongyle eggs were observed and graded under a microscope, and expressed as $1+, 2+, 3+$ and $4+$. Black bars represent strongyle egg-positive cattle. White bars represent strongyle egg-positive goats.

Results of univariable analysis by Chi-square test showed that production purpose $\left(\chi^{2}=9.873, p=0.002\right)$, farm management $\left(\chi^{2}=4.377, p=0.036\right)$, deworming interval $\left(\chi^{2}=14.901, p<0.001\right)$ and flocks $\left(\chi^{2}=34.499, p<0.001\right)$ were significantly associated with the risk of strongyle infection in cattle (Table 3$)$, whereas gender $\left(\chi^{2}=11.456, p<0.001\right)$, oral mucous membrane color $\left(\chi^{2}=11.848, p=0.003\right)$, production purpose $\left(\chi^{2}=20.052\right.$, $p<0.001)$, farm management $\left(\chi^{2}=11.456, p<0.001\right)$, deworming interval $\left(\chi^{2}=11.456\right.$, $p<0.001)$ and flocks $\left(\chi^{2}=32.197, p<0.001\right)$ were significantly associated with the risk of strongyle infection in goats (Table 4 ).

Multivariable logistic regression analysis (Table 5) further showed that dairy cattle (OR 5.069; 95\% CI: 1.461-17.595; $p=0.011$ ) were associated with an increased odds of strongyle infection, i.e., dairy cattle were 5.1 times more likely to be infected with strongyle than meat cattle. Male goats (OR 0.189; 95\% CI: 0.051-0.702; $p=0.013$ ), and dairy goats (OR 0.108; 95\% CI: 0.032-0.371; $p<0.001)$ were associated with decreased odds of strongyle infection. Thus, female goats were 5.3 times (95\% CI: 1.42-19.52) more likely to be infected with strongyle than male goats; and meat goats were 9.3 times (95\% CI: 2.69-31.69) more likely to be infected with strongyle than dairy goats. 
Table 5. Multiple logistic regression of risk factors associated with strongyle infection in cattle and goats.

\begin{tabular}{ccccccc}
\hline Risk Factor & Coefficient & SE & z-Score & $p$-Value & AOR & 95\% CI \\
\hline $\begin{array}{c}\text { Cattle } \\
\text { Production purpose }\end{array}$ & 1.6232 & 0.6349 & 2.556 & 0.011 & 5.069 & $1.461-17.595$ \\
Goat & & & & & & \\
Gender & -1.6625 & 0.6679 & -2.489 & 0.013 & 0.189 & $0.051-0.702$ \\
Production purpose & -2.2241 & 0.6286 & -3.538 & $<0.001$ & 0.108 & $0.032-0.371$ \\
\hline
\end{tabular}

\subsection{Molecular Identification of Gastrointestinal Strongyle in Cattle and Goat Feces}

To describe the strongyle species circulated in the study area, PCR using primers specific to genomic $5.8 \mathrm{~S}$ and $28 \mathrm{~S}$ ribosomal DNA sequences flanking the ITS2 region (primers Strongyle F2/R3) was performed on randomly selected strongyle egg-positive fecal samples (24 samples from cattle and 24 samples from goats). PCR products of the expected sizes were subjected to nucleotide sequencing. The retrieved DNA sequences were analyzed by a BLAST search of the NCBI database, and ITS2 region sequences were compared with reference strongyle sequences, and a phylogenetic tree was then created (Figure 4). We were able to retrieve six strongyle sequences from cattle and eight sequences from goats using nucleotide sequencing. These were from four strongyle genera, Cooperia (six sequences from cattle), Haemonchus (six sequences from goats), Oesophagostomum (one sequence from goats), and Trichostrongylus spp. (one sequence from goats). Phylogenetic analysis suggested the presence of $C$. spatulata or C. punctate (100\% sequence identity), $H$. contortus ( $>98 \%$ sequence identity), Oe. columbianum ( $99 \%$ sequence identity), and T. colubriformis ( $100 \%$ sequence identity).

During sequence analysis, we noticed that some sequences showed a mix of nucleotides in the sequencing chromatogram, suggesting that a number of sequence variants were co-amplified by the primers. Subsequently, in-house semi-nested PCR primers specific to each of the four strongyle genera were used to detect the species involved in the strongyle coinfections (Table 1). Using nested PCR, strongyle genera can be identified in $79.2 \%(19 / 24)$ of cattle samples and $100 \%(24 / 24)$ of goat samples (Table 6). All four strongyles were detected in both cattle and goat feces. In cattle samples, Cooperia $(70.8 \%)$ was found to be the most abundant, followed by Trichostrongylus (45.8\%), Oesophagostomum $(29.2 \%)$, and Haemonchus spp. (16.7\%). In goat samples, Haemonchus was detected in all 24 samples $(100 \%)$, followed by Trichostrongylus $(91.7 \%)$, Oesophagostomum $(37.5 \%)$, and Cooperia spp. (37.5\%). Our investigation in 24 sampling specimens suggested that the majority of samples from cattle $(58.3 \%)$ and goats $(95.8 \%)$ were infected by at least two strongyle species.

Table 6. Strongyle genera identified in this study using semi-nested PCR.

\begin{tabular}{ccc}
\hline \multirow{2}{*}{ Strongyle Infection } & \multicolumn{2}{c}{ No. of Strongyle Detected (\%) } \\
\cline { 2 - 3 } & Cattle $(\boldsymbol{n = 2 4 )}$ & Goat $(\boldsymbol{n}=\mathbf{2 4})$ \\
\hline Strongyle detection by PCR & $19(79.2)$ & $24(100)$ \\
Cooperia spp. & $17(70.8)$ & $9(37.5)$ \\
Haemonchus spp. & $4(16.7)$ & $24(100)$ \\
Oesophagostomum spp. & $7(29.2)$ & $9(37.5)$ \\
Trichostrongylus spp. & $11(45.8)$ & $22(91.7)$ \\
\hline Single infection & $5(20.8)$ & $1(4.2)$ \\
Cooperia spp. & $4(16.7)$ & - \\
Haemonchus spp. & - & $1(4.2)$ \\
Oesophagostomum spp. & $1(4.2)$ & - \\
\hline Coinfection & $14(58.3)$ & $23(95.8)$ \\
C + H & $2(8.3)$ & $1(4.2)$ \\
\hline
\end{tabular}


Table 6. Cont.

\begin{tabular}{ccc}
\hline \multirow{2}{*}{ Strongyle Infection } & \multicolumn{2}{c}{ No. of Strongyle Detected (\%) } \\
\cline { 2 - 3 } & Cattle $(\boldsymbol{n = 2 4 )}$ & Goat $(\boldsymbol{n}=\mathbf{2 4})$ \\
\hline $\mathrm{C}+\mathrm{O}$ & $1(4.2)$ & - \\
$\mathrm{C}+\mathrm{T}$ & $4(16.7)$ & - \\
$\mathrm{H}+\mathrm{T}$ & - & $9(37.5)$ \\
$\mathrm{O}+\mathrm{T}$ & $1(4.2)$ & - \\
$\mathrm{C}+\mathrm{H}+\mathrm{T}$ & $2(8.3)$ & $4(16.7)$ \\
$\mathrm{C}+\mathrm{O}+\mathrm{T}$ & $4(16.7)$ & - \\
$\mathrm{H}+\mathrm{O}+\mathrm{T}$ & - & $5(20.8)$ \\
$\mathrm{C}+\mathrm{H}+\mathrm{O}+\mathrm{T}$ & - & $4(16.7)$ \\
\hline
\end{tabular}

C, Cooperia spp.; H, Haemonchus spp.; O, Oesophagostomum spp.; T, Trichostrongylus spp.

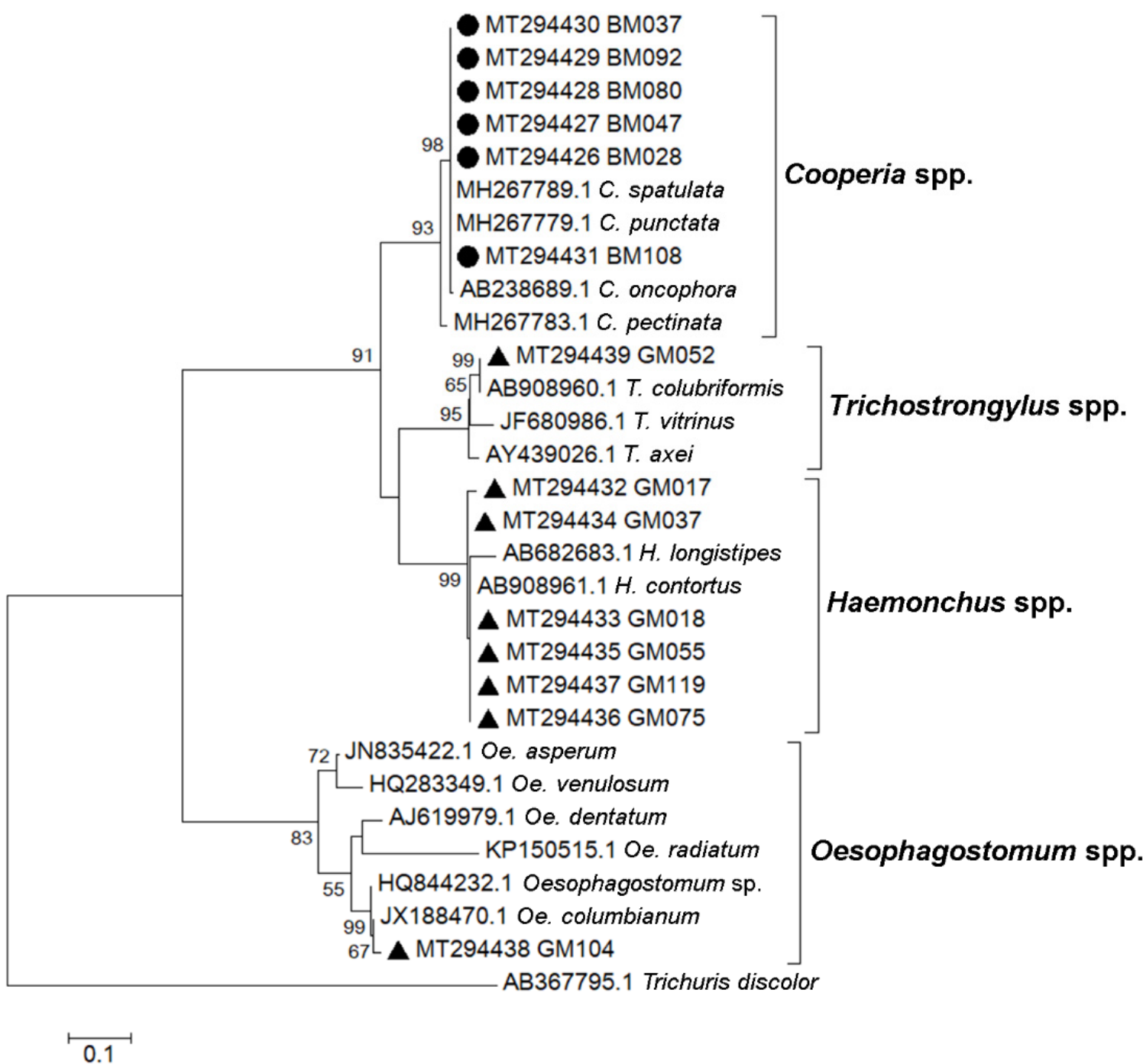

Figure 4. Phylogenetic analysis of ITS2 nucleotide sequences. The tree was constructed using the maximum likelihood method with a bootstrap value of 1000 . Black circles represent strongyle sequences from cattle feces, and black triangles represent strongyle sequences from goat feces. Accession numbers of sequences obtained in this study and of reference nematode sequences are indicated. Bootstrap values of $\geq 50$ are shown at the nodes. The bar represents nucleotide substitutions per site. T. discolor sequence was used as an outgroup. 


\section{Discussion}

Cattle and goats are the main livestock in Thailand and major sources of meat and milk. For the farmers who raise them, infectious diseases that affect animal health and productivity, including bacterial, viral and parasitic (helminth and protozoa) infections, are of concern. It is important to continuously survey for pathogen prevalence, variations, and drug resistance in order to maintain effective intervention strategies within livestock health management. Although a single time point sample collection in a cross-sectional study could limit the sensitivity of parasite detection leading to underestimating of a true parasite prevalence, it provides an accessible and convenient source of up-to-date findings when longitudinal or experimental studies are rarely available [10]. In Thailand, the problem of gastrointestinal helminthic infection in livestock ruminants was well known $[6,7,22]$, but published data to demonstrate its current prevalence was limited. As described above, we performed a cross-sectional epidemiological study of gastrointestinal helminths, especially strongyle nematodes, in cattle and goats in Kanchanaburi Province, which is situated in the western part of Thailand. The area is home to an important part of the livestock industry in Thailand, with many cattle and goat farms. By investigating fecal specimens from 11 cattle farms $(n=157)$ and nine goat farms $(n=117)$, microscopic examination revealed the rates of gastrointestinal helminthic infections in cattle $(35.7 \%)$ and goats $(88 \%)$, with the most commonly observed eggs being those of strongyle nematodes. Eggs of Strongyloides spp., Trichuris spp., Capillaria spp., Paramphistomum spp., and Moniezia spp. were also detected but in lower amounts compared with strongyle eggs.

A survey from Nan Province in the northern part of Thailand, in 2006, showed 61\% overall prevalence of gastrointestinal parasitic infections in beef cattle, with rumen flukes $(28 \%)$ and strongyles (27\%) being the most common parasites [6]. In comparison, we reported $35.7 \%$ overall GI helminth infection in cattle, with strongyles $(28.7 \%)$ as a majority followed by the rumen fluke Paramphistomum spp. (10.2\%). The lower parasite infection rate observed in our study could be due to a sole focus on gastrointestinal helminthic infection, whereas in the previous study both helminths and protozoa were included. Geographical location and climate have also been reported as factors that possibly affect the prevalence and type of parasitic infection in cattle in Thailand $[6,22,23]$. Kanchanaburi Province is in the western central part of the country whereas Nan Province is in the northern part. The climates in these areas are generally different. For the type of helminths detected, although a comparable infection rate of strongyles was observed, the rate of rumen fluke infection in our study was lower than that observed in Nan Province. A higher prevalence of Paramphistomum spp. (25.4\%) was also reported in beef cattle from Phayao Province located close to Nan, in 2019 [23]. The difference in fluke prevalence could be due to the distribution of parasite intermediate hosts, which may vary in different regions of Thailand. For goats, there was a limited report of helminth prevalence from Thailand, even less than that reported in cattle. However, the high individual prevalence $(88 \%)$ and herd prevalence $(100 \%)$ of gastrointestinal helminth eggs observed in this study concurs with previous findings in Nakhon Pathom Province in western central Thailand in 2012, which reported $100 \%$ herd prevalence and $79.5 \%$ individual prevalence of intestinal parasites in goats with strongyle nematodes as the most common parasite found in all positive samples [7]. Strongyles have been the most abundant gastrointestinal parasite infecting livestock in studies carried out since 2003 in countries in various regions such as Lao PDR, Malaysia, Philippines, Pakistan, India, Sudan and Germany. The reported prevalence of strongyles ranged from $11.2-36.0 \%$ in cattle and $9.3-96.2 \%$ in goats $[3,4,8-10,12,24]$. In those studies, Strongyloides spp., Trichuris spp., Capillaria spp., Paramphistomum spp., Fasciola spp., Toxocara spp. and Moniezia spp. were also reported. Besides, coccidia protozoa especially Eimeria spp. were commonly found in cattle and goat feces, with a wide prevalence that ranged from $2 \%$ to $100 \%[6,10,24-26]$. Other protozoa including Entamoeba spp., Blastocystis spp., Balantidium spp., Isospora spp., Cryptosporidium spp. and Giardia spp. were also reported $[7,26,27]$. In our study, we detected the presence of coccidia oocysts in some of the cattle and goat fecal samples, but since it was not our focus, we 
did not subject them to further analysis. Altogether, these findings demonstrate that, for almost two decades, helminth infections, especially strongyle infections, have been a major problem in livestock in Thailand and other countries. Geographical location and climate could be factors that affect the parasites' prevalence, together with other animal and farm management factors that differ in different areas. Concerned with the high prevalence and impact of strongyle infection in livestock ruminants, we focused our analysis mainly on strongyle nematodes.

Multivariate analysis showed that the production purpose (dairy or meat) affected the risk of strongyle infection in cattle and goats in Kanchanaburi. Dairy cattle showed a higher risk of strongyle infection than meat cattle, whereas meat goats showed a higher risk of strongyle infection than dairy goats. The opposite results observed in cattle and goats could be explained by the connection of factors subjected to the analysis. In multivariate analysis, if factors were associated with the others, only one factor with the most significance will be presented. While the production purpose was only shown to be significant in multivariate analysis, it was associated with farm management and deworming intervals. All of these three factors were found to be significantly associated with the risk of strongyle infection in both cattle and goats, as shown by the univariate analysis. In fact, it was found in the sampling groups that most of the dairy cattle were housed in intensive management farms with more than six months deworming interval; and most of the meat goats were kept in extensive management farms with more than six months deworming interval, suggesting the important of deworming practice as the factor that determines the risk of strongyle infection. Proper anthelmintic drug treatment using the correct dose, frequency, and route of administration is vital. A single treatment each year was not effective for preventing infection, especially re-infection, which can occur rapidly after treatment [28]. The deworming program of $\geq 6$ months interval implemented in the dairy cattle and meat goat farms was shown to be insufficient. Three months or less deworming intervals have been suggested for efficient reduction of the risk of intestinal parasitic infection in goats by lowering of parasite eggs and larvae present in the animals' environment [7]. The lack of routine deworming in the dairy cattle may be due to a low level of worm burden that did not cause apparently severe effects to the body condition or health status of the animals, making the farmers unconcerned about the helminthic infection or importance of deworming. Collaborations between the Department of Livestock Development and farmers are needed for the management of effective health care programs that include deworming in livestock farms. An education program should also be available to farm owners to provide basic information about anthelmintic drugs and the effects of parasitic infections on animal production, and to emphasize the importance of health management. Routine surveillance and monitoring of the efficacy of the control programs should be implemented and the infected animals should be promptly treated to reduce the parasitic burden and transmission. Group housing could be another factor that facilitates the parasite transmission, as previously reported [7]. Dairy cattle in the present study were housed intensively in the same pen, meaning that parasitic transmission could occur if there was an infected animal in the herd. The source of infection could be from food and roughage that were used for the animal feeding. Group housing was also used for the meat goats. Although the animals were released to graze freely during the day, they were housed together indoors at night. Free-grazing and sharing of a grazing pasture were shown to be a risk factor for gastrointestinal helminthic infection [6,9]. In addition, it was reported that dairy goat farmers were more concerned about breeding selection and usually practice an appropriate deworming program to maintain good animal health [7]. Sex has been reported as a factor that influences the prevalence of parasitic infection $[9,25]$. In some reports, males were associated with increasing risk of parasitism due to their free grazing practice compared with females which are kept in stall feeding during pregnancy [29,30]. During pregnancy, female animals could also be prone to parasitic infections due to stress and decreased immunity [9]. In our study, female goats were found to be associated with an increased risk of strongyle infection compared to male goats. However, the 
number of males ( $n=18)$ subjected to the study was less than the females $(n=99)$ and the pregnancy status of the female goats was unknown. Strongyle nematodes are well known to cause gastrointestinal parasitism leading to health problems and decrease in animal productions in small ruminants. Thus, Haemonchus contortus, a highly pathogenic blood feeding nematode, has been reported to cause anemia in goats and sheep, which can lead to death in case of heavy infection [14]. Although a high strongyle infection rate and egg burden were detected by microscopy, and Haemonchus spp. were detected in all selected egg-positive samples $(n=24)$ from goats by molecular techniques, we did not statistically observe an association between strongyle infection and anemia parameters (oral mucosa color and PCV) by multivariable analysis in the cattle and goats. This was possibly due to mild infections; the animals enrolled in this study were normal. It was shown in the study from Malaysia that PCV of $\leq 22 \%$ (anemia) was associated with increased strongyle egg counts of approximately 1000 eggs per gram (EPG) of feces, determined by McMaster egg count technique, compared to approximately 700 EPG in non-anemic goats [25]. In this study, an egg grading $(+1$ to +4$)$ was performed to semi-quantitatively estimate the strongyle egg burden, not the absolute egg count, therefore we could not relate the infection to egg numbers. Furthermore, oral mucosa color was examined in both cattle and goats for sign of anemia which could be a result of various health conditions such as blood loss anemia, helminth infection or malnutrition. In sheep and goats, FAMACHA eye scoring is practically used for determining anemia status associated with haemonchosis [31]. A pallor of eye mucosa suggests an infection with the blood-sucking Haemonchus and further anthelmintic treatment. We note these points and suggest that McMaster egg count technique and FAMACHA eye scoring should be applied in a further study.

Generally, strongyle species cannot be differentiated by examining their eggs; instead, coproculture followed by third-stage larval identification is required, which is time-consuming and requires an experienced examiner. Therefore, relatively rapid, uncomplicated, and cost-effective PCR methods are becoming very useful for identifying and confirming morphological diagnoses [3,4]. PCR and DNA sequencing facilitates helminth identification to the genus or species level and allows the identification of multiple strongyle infections that are difficult to differentiate under the microscope [32]. Although the strongyle species identification may unnecessary for treatment management, the information on strongyle types that infect animals provides an understanding of helminth epidemiology, population biology and anthelmintic treatment effectiveness; all of these are important for planning a helminth control program [4]. In this study, we determined species of strongyle nematodes circulating in cattle and goats in the study area, by DNA sequencing of the ITS2 gene and we identified four genera, Cooperia spp., Haemonchus spp., Trichostrongylus spp. and Oesophagostomum spp. These strongyles have been identified, by coproculture or molecular methods, and reported to be common infections of livestock $[3,4,8,9,12]$. Haemonchus spp. were found to be a major strongyle genus infecting goats (25-93\% in goat, and 37\% in cattle). Trichostrongylus spp. (6-76\% in goats, and $16-48 \%$ in cattle) and Oesophagostomum spp. (2-24\% in goats, and $6-16 \%$ in cattle) were commonly found in goats and cattle. Cooperia spp. were mainly found in cattle (prevalence $8-18 \%)$. Their prevalence reported in goats was limited (10\%). Furthermore, we used in-house-designed semi-nested PCR with primers specific to the ITS2 region to differentiate the four strongyle genera according to variations in the PCR product sizes. Our results were in agreement with the previous studies. Haemonchus spp. were abundant in goats $(100 \%)$, whereas Cooperia spp. were mainly found in cattle $(70.8 \%$ in cattle, and $37.5 \%$ in goats). Trichostrongylus spp. ( $91.7 \%$ in goats, and $45.8 \%$ in cattle) and Oesophagostomum spp. (37.5\% in goats, and $29.2 \%$ in cattle) were common in both animals. Polyparasitism has been commonly observed in cattle and goats. Multiple infections of different helminth or protozoan species, as well as mixed infections of helminths and protozoa have been reported $[25-27,33,34]$. The rates of mixed infection found in previous reports varied from $10 \%$ to more than $60 \%$, suggesting that in some settings, polyparasitism was more common than monoparasitism. We observed in our study that $14.3 \%$ and $35.9 \%$ of helminth 
egg-positive samples from cattle and goats, respectively, contained more than one kind of helminth egg. The rate of polyparasitism could be higher if protozoa were included in our examination. Using nested PCR, coinfections with different strongyle genera were observed in the majority of the tested samples, suggesting that multiple infection with different strongyle genera in one host was more common than single strongyle infection, in both cattle and goats. Although all strongyle infections can be treated by the same anthelmintic drug, it has become advantageous to know the coinfection status because there were reports showing that coinfections of particular parasites can affect disease severity or animal product quality. Coinfections of Trichostrongylus spp. and Haemonchus spp., or of these two with other parasites, were shown to impact wool and milk production and health status of small ruminants $[35,36]$. Haemonchus spp. and Trichostrongylus spp. coinfection were found to predominate in goats compared to the other coinfections in our study. Compared with Haemonchus spp. which are highly concerning because they cause severe disease and anthelminthic resistance, Trichostrongylus spp. cause a less severe disease in ruminants [37,38]. However, it was reported in Malaysia to be the second predominant strongyle species after $H$. contortus; and its prevalence was recently found to increase in small ruminants [4]. In our study we found that $91.7 \%$ and $45.8 \%$ of goat and cattle samples, respectively, were positive for Trichostrongylus spp., supporting the suggestion in the previous study that this genus should be viewed with concern as the emerging strongyle species, warranting further monitoring. However, it has to be noted that the samples used for strongyle genus identification in our study were randomly selected from egg-positive samples; therefore, the number reported was not a population prevalence and cannot be absolutely compared with the prevalence obtained in other studies.

\section{Conclusions}

Using microscopic examination, we determined the infection rate of strongyle nematodes and other helminths in cattle and goats in Kanchanaburi Province, Thailand. Molecular identification and sequence analysis of strongyles demonstrated the distribution of the genera Cooperia, Oesophagostomum, Haemonchus, and Trichostrongylus. Most of the animals had multiple infections with different strongyle species. The high rate of strongyle infection in dairy cattle and meat goats suggests the ineffectiveness of the current deworming programs in the area. Overall, the results add recent information about helminths, especially strongyle nematode infection, and related risk factors to the field of epidemiological helminthology. It is of great importance to survey and monitor parasitic prevalence and genetic changes to prevent future outbreaks and drug resistance. Molecular methods such as PCR detection have shown their potential for epidemiological study by improving the diagnosis and identification of strongyle species. Education regarding zoonotic diseases and prevention and control should also be provided, especially for those in regular contact with livestock.

Supplementary Materials: The following are available online at https:/ /www.mdpi.com/article/10.3 390/vetsci8120324/s1, Figure S1: Alignments of strongyle genus-specific primers with sequences of Cooperia spp. (C), Haemonchus spp. (H), Oesophagostomum spp. (O), and Trichostrongylus spp. (T), Figure S2: Specific amplification of ITS-2 gene fragments with strongyle genus-specific primers.

Author Contributions: Conceptualization, N.I. and N.K.; validation, N.I. and N.K.; formal analysis, N.I., C.R. and N.K.; investigation, N.I., J.T. and S.T.; writing-original draft preparation, N.I. and N.K.; writing — review and editing, J.T., S.T., P.A., C.R., P.M. and N.K.; visualization, N.I. and N.K.; supervision, P.A. and P.M.; funding acquisition, N.K. All authors have read and agreed to the published version of the manuscript.

Funding: This research was funded by a Mahidol University research grant and a Thailand One Health University Network research grant. 
Institutional Review Board Statement: The use of animals in this study was approved by the Animal Care and Use Committee of the Faculty of Tropical Medicine (FTM-ACUC), Mahidol University (approval number: FTM-ACUC 009/2016). The animal study was conducted in accordance to the Ethical Principles and Guidelines for the Use of Animals for Scientific Purposes, the National Research Council of Thailand (NRCT).

Informed Consent Statement: Not applicable.

Data Availability Statement: Nucleotide sequences of partial ITS2 have been submitted to the GenBank database. Accession numbers are provided in the materials and methods section, and in Figure 4.

Acknowledgments: This work was supported by a Mahidol University research grant and a Thailand One Health University Network research grant. We thank the Faculty of Tropical Medicine, Mahidol University, for supporting the cost of English editing. We thank the staff of the Livestock and Wildlife Hospital, Faculty of Veterinary Sciences, Mahidol University, for assistance in sample collection.

Conflicts of Interest: The authors declare no conflict of interest. The funders had no role in the design of the study; in the collection, analyses, or interpretation of data; in the writing of the manuscript, or in the decision to publish the results.

\section{References}

1. Lefevre, P.-C.; Blancou, J.; Chermette, R. Infectious and Parasitic Diseases of Livestock; Lavoisier: Paris, France, 2010.

2. Roeber, F.; Jex, A.R.; Gasser, R.B. Next-generation molecular-diagnostic tools for gastrointestinal nematodes of livestock, with an emphasis on small ruminants. Adv. Parasitol. 2013, 83, 267-333. [CrossRef]

3. Sato, M.O.; Sato, M.; Chaisiri, K.; Maipanich, W.; Yoonuan, T.; Sanguankiat, S.; Pongvongsa, T.; Boupha, B.; Moji, K.; Waikagul, J. Nematode infection among ruminants in monsoon climate (Ban-Lahanam, Lao PDR) and its role as food-borne zoonosis. Rev. Bras. Parasitol. Vet. 2014, 23, 80-84. [CrossRef]

4. Tan, T.K.; Panchadcharam, C.; Low, V.L.; Lee, S.C.; Ngui, R.; Sharma, R.S.; AL Lim, Y. Co-infection of Haemonchus contortus and Trichostrongylus spp. among livestock in Malaysia as revealed by amplification and sequencing of the internal transcribed spacer II DNA region. BMC Vet. Res. 2014, 10, 38. [CrossRef]

5. Puspitasari, S.; Farajallah, A.; Sulistiawati, E.; Muladno. Effectiveness of ivermectin and albendazole against Haemonchus contortus in sheep in West Java, Indonesia. Trop. Life Sci. Res. 2016, 27, 135-144.

6. Kaewthamasorn, M.; Wongsamee, S. A preliminary survey of gastrointestinal and haemoparasites of beef cattle in the tropical livestock farming system in Nan Province, northern Thailand. Parasitol. Res. 2006, 99, 306-308. [CrossRef]

7. Ratanapob, N.; Arunvipas, P.; Kasemsuwan, S.; Phimpraphai, W.; Panneum, S. Prevalence and risk factors for intestinal parasite infection in goats raised in Nakhon Pathom Province, Thailand. Trop. Anim. Health Prod. 2011, 44, 741-745. [CrossRef]

8. Rupa, A.P.M.; Portugaliza, H.P. Prevalence and risk factors associated with gastrointestinal nematode infection in goats raised in Baybay city, Leyte, Philippines. Vet. World 2016, 9, 728-734. [CrossRef]

9. Khan, M.N.; Sajid, M.S.; Iqbal, Z.; Hussain, A. Gastrointestinal helminthiasis: Prevalence and associated determinants in domestic ruminants of district Toba Tek Singh, Punjab, Pakistan. Parasitol. Res. 2010, 107, 787-794. [CrossRef]

10. Raue, K.; Heuer, L.; Böhm, C.; Wolken, S.; Epe, C.; Strube, C. 10-year parasitological examination results (2003 to 2012 ) of faecal samples from horses, ruminants, pigs, dogs, cats, rabbits and hedgehogs. Parasitol. Res. 2017, 116, 3315-3330. [CrossRef]

11. Sun, P.; Wronski, T.; Bariyanga, J.D.; Apio, A. Gastro-intestinal parasite infections of Ankole cattle in an unhealthy landscape: An assessment of ecological predictors. Vet. Parasitol. 2018, 252, 107-116. [CrossRef]

12. Mohammedsalih, K.M.; Khalafalla, A.; Bashar, A.; Abakar, A.; Hessain, A.; Juma, F.-R.; Coles, G.; Krücken, J.; Von SamsonHimmelstjerna, G. Epidemiology of strongyle nematode infections and first report of benzimidazole resistance in Haemonchus contortus in goats in South Darfur State, Sudan. BMC Vet. Res. 2019, 15, 184. [CrossRef] [PubMed]

13. Dey, A.R.; Begum, N.; Anisuzzaman; Alim, A.; Alam, M.Z. Multiple anthelmintic resistance in gastrointestinal nematodes of small ruminants in Bangladesh. Parasitol. Int. 2020, 77, 102105. [CrossRef]

14. Selemon, M. Review on control of Haemonchus contortus in sheep and goat. J. Vet. Med. Res. 2018, 5, 1139.

15. Babják, M.; Königová, A.; Dolinská, M.U.; Vadlejch, J.; Várady, M. Anthelmintic resistance in goat herds-In vivo versus in vitro detection methods. Vet. Parasitol. 2018, 254, 10-14. [CrossRef]

16. Torres-Acosta, J.; Hoste, H. Alternative or improved methods to limit gastro-intestinal parasitism in grazing sheep and goats. Small Rumin. Res. 2008, 77, 159-173. [CrossRef]

17. Nguyen, T.; Le, Q.; Huynh, V.; Nguyen, S.; Vu-Khac, H.; Nguyen, T. The development of PCR methodology for the identification of species of the tapeworm Moniezia from cattle, goats and sheep in central Vietnam. J. Helminthol. 2011, 86, 426-429. [CrossRef]

18. Shu, F.-F.; Lv, R.-Q.; Zhang, Y.-F.; Duan, G.; Wu, D.-Y.; Li, B.-F.; Yang, J.-F.; Zou, F.-C. Characterization of Fasciola samples by ITS of rDNA sequences revealed the existence of Fasciola hepatica and Fasciola gigantica in Yunnan Province, China. J. Parasitol. 2012, 98, 889-890. [CrossRef]

19. Jackson, P.; Cockcroft, P. Clinical Examination of Farm Animals; Blackwell Science Ltd.: Oxford, UK, 2002. 
20. Edmonson, A.J.; Lean, I.J.; Weaver, L.D.; Farver, T.; Webster, G. A body condition scoring chart for Holstein dairy cows. J. Dairy Sci. 1989, 72, 68-78. [CrossRef]

21. Ghosh, C.; Datta, S.; Mandal, D.; Das, A.; Roy, D.; Roy, A.; Tudu, N. Body condition scoring in goat: Impact and significance. J. Entomol. Zool. Stud. 2019, 7, 554-560.

22. Chompoochan, T.; Prasitiratana, P.; Nakamura, Y. Preliminary study of nematode infections of cattle in the six provinces of Thailand in the dry season. J. Vet. Med. Sci. 1998, 60, 527-529. [CrossRef]

23. Japa, O.; Siriwechviriya, P.; Prakhammin, K. Occurrence of fluke infection in beef cattle around Phayao Lake, Phayao, Thailand. Vet. World 2020, 13, 334-337. [CrossRef]

24. Murthy, G.S.S.; Rao, P.V. Prevalence of gastro intestinal parasites in ruminants and poultry in Telangana region of Andhra Pradesh. J. Parasit. Dis. 2012, 38, 190-192. [CrossRef]

25. Paul, B.T.; Jesse, F.F.A.; Chung, E.L.T.; Che'Amat, A.; Mohd Lila, M.A. Risk factors and severity of gastrointestinal parasites in selected small ruminants from Malaysia. Vet. Sci. 2020, 7, 208. [CrossRef]

26. Hastutiek, P.; Yuniarti, W.M.; Djaeri, M.; Lastuti, N.D.R.; Suprihati, E.; Suwanti, L.T. Prevalence and diversity of gastrointestinal protozoa in Madura cattle at Bangkalan Regency, East Java, Indonesia. Vet. World 2019, 12, 198-204. [CrossRef]

27. Hassan, N.M.F.; Farag, T.K.; Abu El Ezz, N.M.T.; Abou-Zeina, H.A.A. Prevalence assessment of gastrointestinal parasitic infections among goats in Giza Governorate, Egypt. Bull. Natl. Res. Cent. 2019, 43, 127. [CrossRef]

28. Yanola, J.; Nachaiwieng, W.; Duangmano, S.; Prasannarong, M.; Somboon, P.; Pornprasert, S. Current prevalence of intestinal parasitic infections and their impact on hematological and nutritional status among Karen hill tribe children in Omkoi District, Chiang Mai Province, Thailand. Acta Trop. 2018, 180, 1-6. [CrossRef]

29. Gulland, F.M.; Fox, M. Epidemiology of nematode infections of Soay sheep (Ovis aries L.) on St Kilda. Parasitology 1992, 105, 481-492. [CrossRef]

30. Raza, M.A.; Iqbal, Z.; Jabbar, A.; Yaseen, M. Point prevalence of gastrointestinal helminthiasis in ruminants in southern Punjab, Pakistan. J. Helminthol. 2007, 81, 323-328. [CrossRef]

31. Van Wyk, J.A.; Bath, G.F. The FAMACHA system for managing haemonchosis in sheep and goats by clinically identifying individual animals for treatment. Vet. Res. 2002, 33, 509-529. [CrossRef]

32. Akkari, H.; Jebali, J.; Gharbi, M.; Mhadhbi, M.; Awadi, S.; Darghouth, M.A. Epidemiological study of sympatric Haemonchus species and genetic characterization of Haemonchus contortus in domestic ruminants in Tunisia. Vet. Parasitol. 2013, 193, 118-125. [CrossRef]

33. Ntonifor, H.; Shei, S.; Ndaleh, N.; Mbunkur, G. Epidemiological studies of gastrointestinal parasitic infections in ruminants in Jakiri, Bui Division, North West Region of Cameroon. J. Vet. Med. Anim. Health 2013, 5, 344-352.

34. Das, M.; Laha, R.; Goswami, A. Gastrointestinal parasitism of goats in hilly region of Meghalaya, India. Vet. World 2017, 10, 81-85. [CrossRef]

35. Steel, J.; Jones, W.; Symons, L. Effects of a concurrent infection of Trichostrongylus colubriformis on the productivity and physiological and metabolic responses of lambs infected with Ostertagia circumcincta. Aust. J. Agric. Res. 1982, 33, 131-140. [CrossRef]

36. Abakar, A.; Amin, E.; Osman, A. The interaction of coccidian and Haemonchus contortus infections in desert lambs. Sudan J. Vet. Res. 2001, 17, 15-26.

37. Delano, M.L.; Mischler, S.A.; Underwood, W.J. Biology and Diseases of Ruminants: Sheep, Goats, and Cattle. In Laboratory Animal Medicine; Elsevier: Amsterdam, The Netherlands, 2002; p. 519.

38. Besier, R.B.; Kahn, L.P.; Sargison, N.D.; Van Wyk, J.A. The Pathophysiology, Ecology and Epidemiology of Haemonchus contortus Infection in Small Ruminants. In Advances in Parasitology; Academic Press: San Diego, CA, USA, 2016; Volume 93, pp. 95-143. 